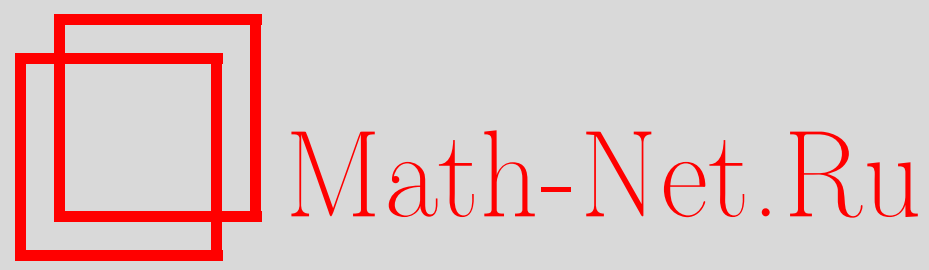

А. С. Шведов, Трехслойная явная разностная схема второго порядка точности для параболических уравнений, Матем. заметки, 1996, том 60, выпуск 5, 751-759

DOI: https://doi.org/10.4213/mzm1886

Использование Общероссийского математического портала MathNet.Ru подразумевает, что вы прочитали и согласны с пользовательским соглашением

http://www . mathnet.ru/rus/agreement

Параметры загрузки:

IP : 54.198 .67 .100

26 апреля 2023 г., 18:10:49

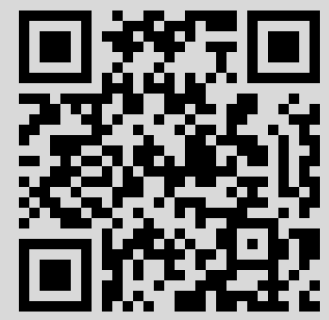




\section{ТРЕХСЛОЙНАЯ ЯВНАЯ РАЗНОСТНАЯ СХЕМА ВТОРОГО ПОРЯДКА ТОЧНОСТИ ДЛЯ ПАРАБОЛИЧЕСКИХ УРАВНЕНИЙ}

А. С. Шведов

\section{§1. Постановка задачи и описание разностной схемы}

Рассмотрим некоторую краевую задачу для уравнения параболического типа

$$
\frac{\partial v}{\partial t}=\sum_{i, j=1}^{d} \frac{\partial}{\partial x_{i}}\left(\sigma_{i j}(x) \frac{\partial v}{\partial x_{j}}\right)+g(x, t) .
$$

Будем считать, что на область $G \times[0, T]$, где ищется решение, на коэффициенты уравнения, на правую часть и на величины на границах наложены условия, гарантирующие однозначную разрешимость краевой задачи и достаточную гладкость решения. Для простоты изложения мы считаем, что коэффициенты $\sigma_{i j}$ не зависят от $t$, хотя это ограничение не является принципиальным.

Если в области $G$ построена сетка (т.е. выбрано $N$ точек, принадлежащих $G$ ), то дифференциальную задачу (1) можно заменить дифференциально-разностной задачей

$$
\frac{d u}{d t}=-A u+f(t)
$$

Здесь $u(t)$ и $f(t)$ при любом $t \in[0, T]$ являются $N$-мерными векторами, матрица $A$ размера $N \times N$ симметрична и ее собственные числа неотрицательны. Вектор $u(0)$ считается заданньм. Требуется, чтобы при измельчении сетки решения задачи (2) сходились к решению задачи (1). Подробнее о связи задач (1) и (2) сказано в 55 .

Работа выполнена при поддержке Российского фонда фундаментальных исследований, грант № 95-01-00489, и Международного научного фонда, гранты N8Q300, JBR100. 
В дальнейшем до $§ 5$ мы будем рассматривать только задачу (2). Задача (2) может возникнуть и независимо от задачи (1), если рассматривается не параболическое уравнение, а система обыкновенных дифференциальных уравнений. Для нас, однако, существенно, что матрица $A$ симметрична и ее собственные значения неотрицательны.

Следующий этап состоит в переходе от дифференциально-разностной задачи (2) к разностной задаче. Пусть выбран временной шаг $\tau>0$. Через $y^{j}$ обозначим $N$-мерньй вектор, являющийся решением разностной задачи для момента времени $j \tau$, где $0 \leqslant j \tau \leqslant T$. Для удобства будем считать, что число $T / \tau$ целое. Через $f^{j}$ обозначим правую часть $f(t)$ при $t=j \tau$. Введем обозначения $\varphi_{0}=\tau f^{j}, \varphi_{1}=\frac{1}{2} \tau f^{j}+\frac{1}{2} \tau f^{j+1}$. Рассмотрим матрицы $B=E+\frac{1}{2} \tau A, B_{1}=E-\frac{1}{2} \tau A$, где через $E$ обозначена единичная матрица размера $N \times N$.

Разностные уравнения для определения $y^{j+1}$ запишем следуюшим образом:

$$
\begin{aligned}
& \bar{y}^{j+1}=y^{j-1}-2 \tau A y^{j}+2 \varphi_{0}, \\
& y^{j+1}=\bar{y}^{j+1}-\omega\left(B \bar{y}^{j+1}-\left(B_{1} y^{j}+\varphi_{1}\right)\right) .
\end{aligned}
$$

О выборе параметра $\omega$ будет сказано ниже. Первое уравнение, в котором определяется $\bar{y}^{j+1}$, - это уравнение разностной схемы Ричардсона. Второе уравнение, в котором определяется $y^{j+1}$, возникает из решения системы линейных уравнений, соответствуюших неявной разностной схеме Крэнка-Никольсон $B z^{j+1}=B_{1} z^{j}+\varphi_{1}$ итерационным методом, но при этом делается всего одна итерация, а в качестве нулевого приближения выбирается $\bar{y}^{j+1}$. (Мы обозначили через $z^{j}$ решение, полученное для момента времени $j \tau$ по разностной схеме Крэнка-Никольсон, чтобы не путать его с решением $y^{j}$, полученным по разностной схеме (3).) Поэтому мы говорим, что разностная схема (3), про которую будет доказано, что при определенном выборе чисел $\omega$ и $\tau$ она устойчива и имеет второй порядок точности, является комбинацией разностных схем Ричардсона и Крэнка-Никольсон.

Будем считать, что числа $\omega$ и $\tau$ удовлетворяют следующим условиям:

$$
\frac{1}{\sqrt{2}+0.75} \leqslant \omega \leqslant 1
$$

и

$$
\tau \leqslant \frac{2}{\Lambda} \min \left(2 \cdot \frac{1-\omega}{\omega}, \frac{1-\omega}{\omega}+0.25\right),
$$

где $\Lambda$ - максимальное собственное число матрицы $A$.

Через $u^{j}$ обозначим решение $u(t)$ дифференциально-разностной задачи $(2)$ при $t=j \tau$. Для $N$-мерного вектора $y=\left(y_{1}, y_{2}, \ldots, y_{N}\right)$ через $\|\cdot\|$ обозначается $L^{2}$-норма:

$$
\|y\|=\left(\frac{1}{N} \sum_{k=1}^{N}\left|y_{k}\right|^{2}\right)^{1 / 2} .
$$


Основным результатом работы является следуюшая теорема.

Теорема 1. При любом $j=2,3, \ldots, T / \tau$

$$
\left\|u^{j}-y^{j}\right\| \leqslant \max \left(\left\|u^{0}-y^{0}\right\|,\left\|u^{1}-y^{1}\right\|\right)+C \cdot j \cdot \tau^{3} .
$$

Константа С не зависит ни от $j$, ни от $\tau$.

ДоКАЗАТЕЛЬСТВо этой теоремы содержится в $§ 2-4$.

Наиболее слабые ограничения на временной шаг $\tau$ получаются при $\omega=1 /(\sqrt{2}+0.75)$. В этом случае утверждение теоремы 1 справедливо при $\tau \leqslant 2 \sqrt{2} / \Lambda$.

Отметим, что похожий метод построения трехслойных явных разностных схем для параболических уравнений использовался в [3]. Подробнее об этих разностных схемах и об их связи с нашей разностной схемой сказано в [4].

\section{§2. Преобразование разностных уравнений}

Заметим, что первое из уравнений (3) можно записать в виде

$$
\bar{y}^{j+1}=y^{j-1}-2\left(B y^{j}-\left(B_{1} y^{j}+\varphi_{0}\right)\right) .
$$

Подставляя это выражение во второе из уравнений $(3)$, получаем

$$
\begin{aligned}
y^{j+1}= & y^{j-1}-2\left(B y^{j}-\left(B_{1} y^{j}+\varphi_{0}\right)\right) \\
& -\omega B\left(y^{j-1}-2\left(B y^{j}-\left(B_{1} y^{j}+\varphi_{0}\right)\right)\right)+\omega\left(B_{1} y^{j}+\varphi_{1}\right) \\
= & (E-\omega B) y^{j-1}-2 B(E-\omega B) y^{j}+(2 E-2 \omega B+\omega E) B_{1} y^{j} \\
& +2(E-\omega B) \varphi_{0}+\omega E \varphi_{1} .
\end{aligned}
$$

Вводя обозначения $Z=E-\omega B, S=-2 B Z+(2 Z+\omega E) B_{1}$, получаем

$$
y^{j+1}=Z y^{j-1}+S y^{j}+2 Z \varphi_{0}+\omega E \varphi_{1} .
$$

Найдем еще одно представление для оператора $S$ :

$$
S=-2 B Z+\left(2 Z+(E-Z) B^{-1}\right) B_{1}=\left(\left(-2 B^{2}+2 B B_{1}-B_{1}\right) Z+B_{1}\right) B^{-1} .
$$

Выражая $B$ и $B_{1}$ через $A$, получаем

$$
S=\frac{E-\frac{1}{2} \tau A-\left(4\left(\frac{1}{2} \tau A\right)^{2}+3\left(\frac{1}{2} \tau A\right)+E\right) Z}{E+\frac{1}{2} \tau A} .
$$

\section{§3. Доказательство устойчивости по начальным данным}

В этом параграфе мы докажем, что если $\omega$ и $\tau$ удовлетворяют условиям (4) и (5), то

$$
\|Z\|+\|S\| \leqslant 1
$$


Здесь через $\|Z\|$ и $\|S\|$ обозначены нормы матриц $Z$ и $S$. Воспользуемся тем фактом, что если $K$ - симметричная матрица размера $N \times N$ и $\varkappa_{1}, \varkappa_{2}, \ldots, \varkappa_{N}-$ ее собственные числа, то

$$
\|K\|=\max _{1 \leqslant i \leqslant N}\left|\varkappa_{i}\right| .
$$

Пусть $\lambda_{1}, \lambda_{2}, \ldots, \lambda_{N}$ - собственные числа матрицы $A$. По условию все эти числа принадлежат отрезку $[0, \Lambda]$. Числа $\xi_{i}=\frac{1}{2} \tau \lambda_{i}, i=1,2, \ldots, N$, являются собственньми числами матрицы $\frac{1}{2} \tau A$.

Определим функции

$$
\mu(\xi)=1-\omega(1+\xi), \quad \nu(\xi)=\frac{1-\left(\xi+\mu(\xi)\left(4 \xi^{2}+3 \xi+1\right)\right)}{1+\xi} .
$$

На основании определения матрицы $Z$ и представления (8) для матрицы $S$ имеем

$$
\|Z\|=\max _{1 \leqslant i \leqslant N}\left|\mu\left(\xi_{i}\right)\right| \quad \text { и } \quad\|S\|=\max _{1 \leqslant i \leqslant N} \mid \nu\left(\xi_{i} \mid,\right.
$$

откуда

$$
\|Z\| \leqslant \max _{\xi \in\left[0, \frac{1}{2} \tau \Lambda\right]}|\mu(\xi)| \quad \text { и } \quad\|S\| \leqslant \max _{\xi \in\left[0, \frac{1}{2} \tau \Lambda\right]}|\nu(\xi)| .
$$

Мы докажем, что если $\omega$ и $\tau$ удовлетворяют условиям (4) и (5), то

$$
\max _{\xi \in\left[0, \frac{1}{2} \tau \Lambda\right]}|\mu(\xi)|+\max _{\xi \in\left[0, \frac{1}{2} \tau \Lambda\right]}|\nu(\xi)|=1,
$$

откуда будет следовать (9).

Из определения функции $\nu(\xi)$ следует, что $\mu(0)+\nu(0)=1$. Поэтому для вьполнения (10) необходимо, чтобы соблюдались условия

$$
\begin{gathered}
\max _{\xi \in\left[0, \frac{1}{2} \tau \Lambda\right]}|\mu(\xi)|=\mu(0), \\
\max _{\xi \in\left[0, \frac{1}{2} \tau \Lambda\right]}|\nu(\xi)|=\nu(0) .
\end{gathered}
$$

Для доказательства соотношений (11) и (12) удобно ввести новое обозначение $x=(1-\omega) / \omega$. Тогда $\mu(\xi)=(x-\xi) /(x+1)$ и условие $(11)$ выполняется в том и только том случае, если

$$
\frac{1}{2} \tau \Lambda \leqslant 2 x
$$

Условие (12) выполняется тогда и только тогда, когда для произвольного $\xi \in\left[0, \frac{1}{2} \tau \Lambda\right]$

$$
-\nu(0) \leqslant \nu(\xi)
$$

И

$$
\nu(\xi) \leqslant \nu(0)
$$


Замечая, что $\nu(0)=1 /(x+1)$, запишем условие (14) в виде

$$
-\frac{1+\xi}{x+1} \leqslant 1-\xi-\frac{x-\xi}{x+1}\left(4 \xi^{2}+3 \xi+1\right)
$$

или

$$
(x-\xi)\left(4 \xi^{2}+3 \xi+1\right) \leqslant(x+2)-\xi x .
$$

Аналогично, условие (15) записывается в виде

$$
(x-\xi)\left(4 \xi^{2}+3 \xi+1\right) \geqslant x-\xi(x+2) .
$$

Таким образом, условие (12) вьполняется в том и только в том случае, если на отрезке $\left[0, \frac{1}{2} \tau \Lambda\right]$ график функции $\zeta=(x-\xi)\left(4 \xi^{2}+3 \xi+1\right)$ лежит между графиками прямых $\zeta=-\xi x+(x+2)$ и $\zeta=-\xi(x+2)+x$. Прежде, чем провести строгое обсуждение условий, при которых график кубической параболы лежит между графиками прямых, отметим, что графики этих функций при трех различных значениях $x$ показаны на рис. $1-3$.

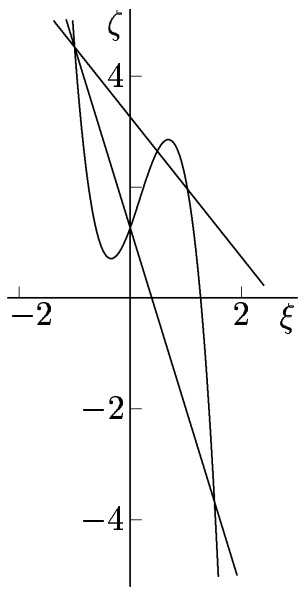

Рис. 1

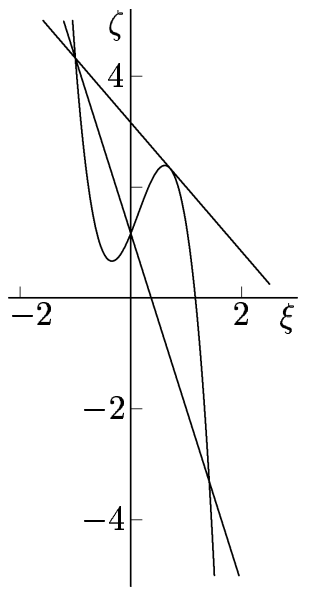

Рис. 2

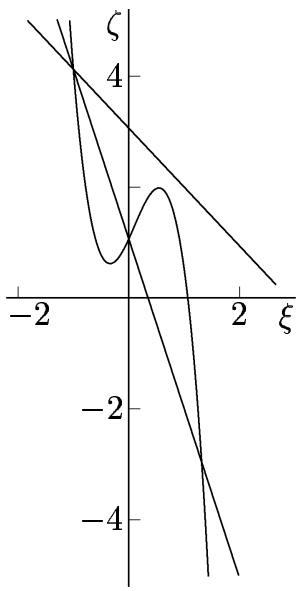

Рис. 3

Графики кубической параболы $\zeta=(x-\xi)\left(4 \xi^{2}+3 \xi+1\right)$ и прямых $\zeta=-\xi x+(x+2)$ и $\zeta=-\xi(x+2)+x$ при $x=\sqrt{2}-0.15$ (рис. 1$)$, при $x=\sqrt{2}-0.25$ (рис. 2), при $x=\sqrt{2}-0.35$ (рис. 3 ).

Сначала обсудим условия, при которых на отрезке $\left[0, \frac{1}{2} \tau \Lambda\right]$ график кубической параболы лежит под графиком верхней прямой. Заметим, что при любом $x$ кубическая парабола пересекается с этой прямой в точке с абсциссой $\xi=-1$. Другие точки пересечения существуют в том и только в том случае, когда уравнение

$$
4 \xi^{2}-(4 x+1) \xi+2=0
$$


имеет действительные корни. При $x \leqslant \sqrt{2}-0.25$ действительных корней у уравнения (18) не больше одного (кубическая парабола при $\xi \geqslant 0$ лежит строго под верхней прямой или касается этой прямой в одной точке) и условие (16) выполнено при любом $\xi \geqslant 0$. При $x>\sqrt{2}-0.25$ действительных корней у уравнения (18) два (кубическая парабола при $\xi \geqslant 0$ имеет две точки пересечения с верхней прямой). Можно показать, что условие (16) нарушается при достаточно малом $\xi$, а наша цель состоит в том, чтобы сделать число $\tau$ (и, следовательно, число $\frac{1}{2} \tau \Lambda$ ) как можно больше. Поэтому будем считать, что выполнено условие $0<x \leqslant \sqrt{2}-0.25$, равносильное условию $1 /(\sqrt{2}+0.75) \leqslant \omega<1$.

Одна точка пересечения кубической параболы с нижней прямой известна $(\xi=0)$. Две другие точки пересечения определяются из квадратного уравнения

$$
4 \xi^{2}-(4 x-3) \xi-(4 x+1)=0
$$

и оказьваются равными $\xi_{1}=-1, \xi_{2}=x+0.25$. Таким образом, условие (17) вьполняется при

$$
\frac{1}{2} \tau \Lambda \leqslant x+0.25 \text {. }
$$

Условия (13) и (20) дают ограничение на временной шаг $\tau$ :

$$
\tau \leqslant \frac{2}{\Lambda} \min (2 x, x+0.25)
$$

совпадающее с (5).

Равенства (11) и (12) доказаны. Вместе с ними доказаны равенство (10) и неравенство (9).

\section{§4. Доказательство сходимости}

Положим

$$
\begin{aligned}
& w_{0}^{j+1}=u^{j-1}-2 \tau A u^{j}+2 \varphi_{0}, \\
& w_{1}^{j+1}=B^{-1} B_{1} u^{j}+B^{-1} \varphi_{1}, \\
& w_{2}^{j+1}=Z u^{j-1}+S u^{j}+2 Z \varphi_{0}+\omega E \varphi_{1} .
\end{aligned}
$$

Из разложения в ряд Тейлора и из (2) получаем

$$
u^{j+1}=u^{j-1}-2 \tau A u^{j}+2 \varphi_{0}+O\left(\tau^{3}\right) .
$$

Отсюда

$$
u^{j+1}=w_{0}^{j+1}+\varepsilon_{0}^{j+1},
$$

где $\left\|\varepsilon_{0}^{j+1}\right\| \leqslant C_{0} \tau^{3}$. Последнеенеравенство справедливо, поскольку $\left\|\varepsilon_{0}^{j+1}\right\|$ не превосходит норму $\varepsilon_{0}^{j+1}$ в метрике $C$.

Еще раз воспользовавшись разложением в ряд Тейлора и (2), получаем

$$
B u^{j+1}=B_{1} u^{j}+\varphi_{1}+O\left(\tau^{3}\right) .
$$


Отсюда

$$
u^{j+1}=w_{1}^{j+1}+B^{-1} \varepsilon_{1}^{j+1},
$$

где $\left\|\varepsilon_{1}^{j+1}\right\| \leqslant C_{1} \tau^{3}$.

Заметим, что

$$
\left\|u^{j+1}-y^{j+1}\right\| \leqslant\left\|u^{j+1}-w_{1}^{j+1}\right\|+\left\|w_{1}^{j+1}-w_{2}^{j+1}\right\|+\left\|w_{2}^{j+1}-y^{j+1}\right\| .
$$

Оценим по отдельности каждое из трех слагаемых правой части последнего неравенства:

$$
\left\|u^{j+1}-w_{1}^{j+1}\right\| \leqslant\left\|B^{-1}\right\| \cdot\left\|\varepsilon_{1}^{j+1}\right\| \leqslant C_{1} \tau^{3},
$$

поскольку

$$
\left\|B^{-1}\right\| \leqslant 1
$$

С учетом (8) имеем

$$
\begin{aligned}
w_{1}^{j+1}-w_{2}^{j+1}= & B^{-1} B_{1} u^{j}+B^{-1} \varphi_{1}-Z u^{j-1}-S u^{j}-2 Z \varphi_{0}-\omega B B^{-1} \varphi_{1} \\
= & B^{-1} B_{1} u^{j}-Z\left(w_{0}^{j+1}+2 \tau A u^{j}-2 \varphi_{0}\right) \\
& -S u^{j}-2 Z \varphi_{0}+Z B^{-1} \varphi_{1} \\
= & B^{-1} B_{1} u^{j}-Z\left(w_{0}^{j+1}+2 \tau A u^{j}\right) \\
& -\left(B_{1}-\left(\tau^{2} A^{2}+\frac{3}{2} \tau A+E\right) Z\right) B^{-1} u^{j}+Z B^{-1} \varphi_{1} \\
= & -Z w_{0}^{j+1}-2 \tau A\left(E+\frac{1}{2} \tau A\right) Z B^{-1} u^{j} \\
& +\left(\tau^{2} A^{2}+\frac{3}{2} \tau A+E\right) Z B^{-1} u^{j}+Z B^{-1} \varphi_{1} \\
= & -Z w_{0}^{j+1}+\left(-\frac{1}{2} \tau A+E\right) Z B^{-1} u^{j}+Z B^{-1} \varphi_{1} \\
= & Z\left(w_{1}^{j+1}-w_{0}^{j+1}\right) .
\end{aligned}
$$

С учетом (21) и (22) получаем

$$
w_{1}^{j+1}-w_{2}^{j+1}=Z\left(\varepsilon_{0}^{j+1}-B^{-1} \varepsilon_{1}^{j+1}\right) .
$$

В силу (9) и (24)

$$
\left\|w_{1}^{j+1}-w_{2}^{j+1}\right\| \leqslant\left(C_{0}+C_{1}\right) \tau^{3} .
$$

Наконец, с учетом (7)

$$
w_{2}^{j+1}-y^{j+1}=Z\left(u^{j-1}-y^{j-1}\right)+S\left(u^{j}-y^{j}\right) .
$$

Имеем

$$
\begin{aligned}
\left\|w_{2}^{j+1}-y^{j+1}\right\| & \leqslant\|Z\| \cdot\left\|u^{j-1}-y^{j-1}\right\|+\|S\| \cdot\left\|u^{j}-y^{j}\right\| \\
& \leqslant(\|Z\|+\|S\|) \max \left(\left\|u^{j-1}-y^{j-1}\right\|,\left\|u^{j}-y^{j}\right\|\right) .
\end{aligned}
$$


В силу (9)

$$
\left\|w_{2}^{j+1}-y^{j+1}\right\| \leqslant \max \left(\left\|u^{j-1}-y^{j-1}\right\|,\left\|u^{j}-y^{j}\right\|\right) .
$$

Собирая вместе оценки $(23),(25)$ и (26), получаем

$$
\left\|u^{j+1}-y^{j+1}\right\| \leqslant \max \left(\left\|u^{j-1}-y^{j-1}\right\|,\left\|u^{j}-y^{j}\right\|\right)+C \tau^{3},
$$

откуда следует (6). Теорема 1 доказана.

\section{§5. О связи задач (1) и (2)}

Пусть сетка состоит из $N$ точек $x^{1}, x^{2}, \ldots, x^{N}$, принадлежащих области $G$. Обозначим через $w$ функцию, определенную на отрезке $[0, T]$, принимающую значения в $N$-мерном пространстве и такую, что $w_{i}(t)=v\left(x^{i}, t\right)$ при любом $i=1,2, \ldots, N$ и при любом $t \in[0, T]$, где $v-$ это решение рассматриваемой краевой задачи для уравнения (1). Будем считать, что задача (2) (точнее, последовательность задач (2)) аппроксимирует задачу (1), т.е., во-первых, функции $u$ и $w$ совпадают при $t=0$, и, во-вторых, функция $w$ является решением системы уравнений

$$
\frac{d w}{d t}=-A w+f(t)+\psi(t)
$$

где $\|\psi(t)\| \rightarrow 0$ при $N \rightarrow \infty$ равномерно на $[0, T]$. Так же, как и в основной части работы, здесь через $\|\cdot\|$ обозначена норма $L^{2}$ для $N$-мерного вектора.

Положим $z(t)=w(t)-u(t)$.

Tеорема 2. $\|z(t)\| \rightarrow 0$ при $N \rightarrow \infty$ равномерно на $[0, T]$.

ДокАЗАТЕЛЬСТВо. $z$ является решением системы уравнений

$$
\frac{d z}{d t}=-A z+\psi(t)
$$

Пусть $X_{1}, X_{2}, \ldots, X_{N}$ - собственные векторы матрицы $A$, т.е. $A X_{k}=\lambda_{k} X_{k}$ при $k=1,2, \ldots, N$. Напомним, что $\lambda_{k} \geqslant 0$ для всех $k$. Будем считать, что векторы $X_{1}, X_{2}, \ldots, X_{N}$ образуют ортонормированный базис $N$-мерного пространства и

$$
\psi(t)=\sum_{k=1}^{N} \psi_{k}(t) X_{k}, \quad z(t)=\sum_{k=1}^{N} z_{k}(t) X_{k} .
$$

Из (27) получаем

$$
\frac{d z_{k}(t)}{d t}=-\lambda_{k} z_{k}(t)+\psi_{k}(t), \quad k=1,2, \ldots, N .
$$


Переходя к новым неизвестным $c_{k}(t)=z_{k}(t) \exp \left(\lambda_{k} t\right)$, получаем для них уравнения

$$
\frac{d c_{k}(t)}{d t}=\psi_{k}(t) \exp \left(\lambda_{k} t\right)
$$

Учитьвая, что $z_{k}(0)=0$, находим

$c_{k}(t)=\int_{0}^{t} \psi_{k}(s) \exp \left(\lambda_{k} s\right) d s \quad$ и $\quad z_{k}(t)=\int_{0}^{t} \psi_{k}(s) \exp \left(-\lambda_{k}(t-s)\right) d s$.

С учетом того, что $\lambda_{k} \geqslant 0$, имеем

$$
\left|z_{k}(t)\right| \leqslant \int_{0}^{t}\left|\psi_{k}(s)\right| d s
$$

Отсюда следует, что

$$
\left|z_{k}(t)\right|^{2} \leqslant t \int_{0}^{t}\left|\psi_{k}(s)\right|^{2} d s
$$

Суммируя последние неравенства по всем $k$, получаем

$$
\|z(t)\|^{2} \leqslant t \int_{0}^{t}\|\psi(s)\|^{2} d s
$$

и из того, что $\|\psi(t)\| \rightarrow 0$ при $N \rightarrow \infty$ равномерно на $[0, T]$, получаем, что $\|z(t)\| \rightarrow 0$ при $N \rightarrow \infty$ равномерно на [0,T]. Теорема 2 доказана.

Приведенные в [4] результаты расчетов двух одномерных краевых задач показьвают, что для данных краевых задач предлагаемая трехслойная явная разностная схема и двухслойная неявная разностная схема Крэнка-Никольсон дают приблизительно одинаковые результаты. Результаты, полученные по двухслойной явной разностной схеме, оказываются значительно хуже.

Институт прикладной математики

Поступило

им. М.В. Келдыша РАН

28.12 .95

\section{СПИСОК ЦИТИРОВАННОЙ ЛИТЕРАТУРЫ}

[1] Richardson L.F. The approximate solution by finite differences of physical problems involving differential equations with an application to the stresses in a masonry dam // Roy. Soc. Philos. Trans. 1910. V. 210A. P. 307-357.

[2] Crank J., Nicolson P. A practical method for numerical integration of solutions of partial differential equations of heat conduction type. I // Proc. Cambridge Philos. Soc. 1947. V. 43. P. 50-67.

[3] Юань Чжао-дин. Некоторые разностные схемы решения первой краевой задачи для линейных дифференциальных уравнений с частными производными. Дисс. ... к. ф.-м.н. М.: МГУ, 1958.

[4] Шведов А.С. Построение трехслойной явной разностной схемы второго порядка точности для параболических уравнений на основе разностных схем Ричардсона и Крэнка-Никольсон. Препринт ИПМ им. М. В. Келдьшша РАН№ 104. M., 1995. 